\title{
Blocking type I interferon signaling enhances T cell recovery and reduces HIV-1 reservoirs
}

\author{
Liang Cheng, Jianping Ma, ${ }^{2}$ Jingyun Li, ${ }^{2}$ Dan Li ${ }^{1,3}$ Guangming Li, ${ }^{1}$ Feng Li, ${ }^{1}$ Qing Zhang ${ }^{1,4}$ Haisheng Yu, ${ }^{2}$ Fumihiko Yasui, ${ }^{1}$ \\ Chaobaihui Ye, ${ }^{1}$ Li-Chung Tsao, ${ }^{1}$ Zhiyuan Hu, ${ }^{1}$ Lishan Su, ${ }^{1,2,5}$ and Liguo Zhang ${ }^{2}$
}

'Lineberger Comprehensive Cancer Center, University of North Carolina at Chapel Hill, Chapel Hill, North Carolina, USA. ${ }^{2}$ Key Laboratory of Infection and Immunity, Institute of Biophysics, Chinese Academy

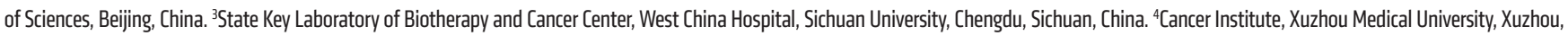
Jiangsu, China. ${ }^{5}$ Department of Microbiology and Immunology, University of North Carolina at Chapel Hill, Chapel Hill, North Carolina, USA.

\begin{abstract}
Despite the efficient suppression of HIV-1 replication that can be achieved with combined antiretroviral therapy (cART), low levels of type I interferon (IFN-I) signaling persist in some individuals. This sustained signaling may impede immune recovery and foster viral persistence. Here we report studies using a monoclonal antibody to block IFN- $\alpha / \beta$ receptor (IFNAR) signaling in humanized mice (hu-mice) that were persistently infected with HIV-1. We discovered that effective cART restored the number of human immune cells in HIV-1-infected hu-mice but did not rescue their immune hyperactivation and dysfunction. IFNAR blockade fully reversed HIV-1-induced immune hyperactivation and rescued anti-HIV-1 immune responses in T cells from HIV-1-infected hu-mice. Finally, we found that IFNAR blockade in the presence of CART reduced the size of HIV-1 reservoirs in lymphoid tissues and delayed HIV-1 rebound after CART cessation in the HIV-1-infected hu-mice. We conclude that low levels of IFN-I signaling contribute to HIV-1-associated immune dysfunction and foster HIV-1 persistence in cARTtreated hosts. Our results suggest that blocking IFNAR may provide a potential strategy to enhance immune recovery and reduce HIV-1 reservoirs in individuals with sustained elevations in IFN-I signaling during suppressive CART.
\end{abstract}

\section{Introduction}

Type I interferons (IFN-I) are critical for controlling virus infections $(1,2)$, but they also contribute to impaired host immunity and virus persistence $(3,4)$. The precise role of IFN-I during chronic HIV-1 infection remains unclear $(5,6)$. HIV-1 infection induces widespread expression of IFN-I and IFN-stimulated genes (ISGs) $(7,8)$. It has been reported that IFN-I can suppress HIV-1 replication in vitro (5), and the major anti-HIV-1 restriction factors are encoded by ISGs (5). In addition, IFN-I has been shown to inhibit early HIV-1 infection in humanized mice (hu-mice) (9) and SIV infection in rhesus macaques in vivo (10). These observations suggest that a robust IFN-I response helps to control or limit initial HIV-1 and SIV infection.

IFN-I has also been implicated in the immunopathogenesis of AIDS during chronic HIV-1 infection $(5,6)$. Studies using nonhuman primate models have documented that sustained IFN-I signaling is associated with pathogenic SIV infection (11-14). IFN-I is induced during the acute phase of SIV infection in both pathogenic

\section{Related Commentary: p. 103}

Authorship note: L. Cheng, J. Ma, and J. Li contributed equally to this work. Note regarding evaluation of this manuscript: Manuscripts authored by scientists associated with Duke University, The University of North Carolina at Chapel Hill, Duke-NUS, and the Sanford-Burnham Medical Research Institute are handled not by members of the editorial board but rather by the science editors, who consult with selected external editors and reviewers.

Conflict of interest: The authors have declared that no conflict of interest exists. Submitted: September 19, 2016; Accepted: October 27, 2016.

Reference information: J Clin Invest. 2017;127(1):269-279. doi:10.1172/JCI90745. (rhesus macaques or pigtail macaques) and nonpathogenic hosts (African green monkeys or sooty mangabeys). However, compared with the nonpathogenic natural SIV infection, pathogenic SIV infection leads to AIDS development, associated with sustained IFN-I signaling (11-14). Furthermore, studies in HIV-1-infected patients indicate that expression of IFN-I and ISGs is correlated with a higher level of viral load, enhanced hyperimmune activation, and faster disease progression $(8,15-17)$. Using the mouse model of lymphocytic choriomeningitis virus persistent infection, it is reported that blocking of IFN-I signaling by IFNAR antibody can reverse immune suppression, restore lymphoid architecture, and accelerate clearance of the virus $(3,4)$.

Administration of exogenous IFN- $\alpha$ can lower HIV-1 burden in HIV-1-infected patients but fail to show a significant benefit in HIV-1 disease progression (6). Interestingly, recent studies report that the administration of IFN- $\alpha$ in HIV-1-monoinfected patients or patients coinfected with HIV-1 and hepatitis C virus (HCV) results in reduction of cell-associated viral RNA and DNA in the blood (18-21). However, other studies in HIV-1-infected patients indicate that persistent expression of ISGs is correlated with higher viral load, enhanced hyperimmune activation, and faster disease progression $(8,15-17)$. In addition, administration of IFN- $\alpha$ to patients also leads to a decrease in $\operatorname{CD} 4 \mathrm{~T}$ cell count $(18,21)$ and enhanced CD8 T cell activation (22) in the blood. Moreover, despite efficient suppression of HIV-1 replication with combined antiretroviral therapy (cART), abnormally elevated IFN-I signaling persists in some patients even under extensive cART $(23,24)$, which may impede the reversion of hyperimmune activation and immune recovery in those immune nonresponder patients (25). 


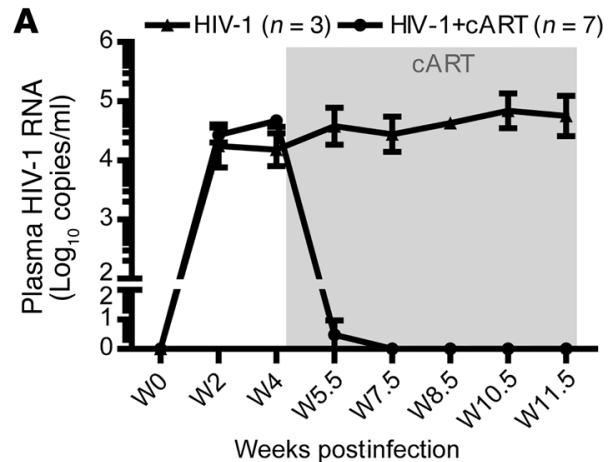

C

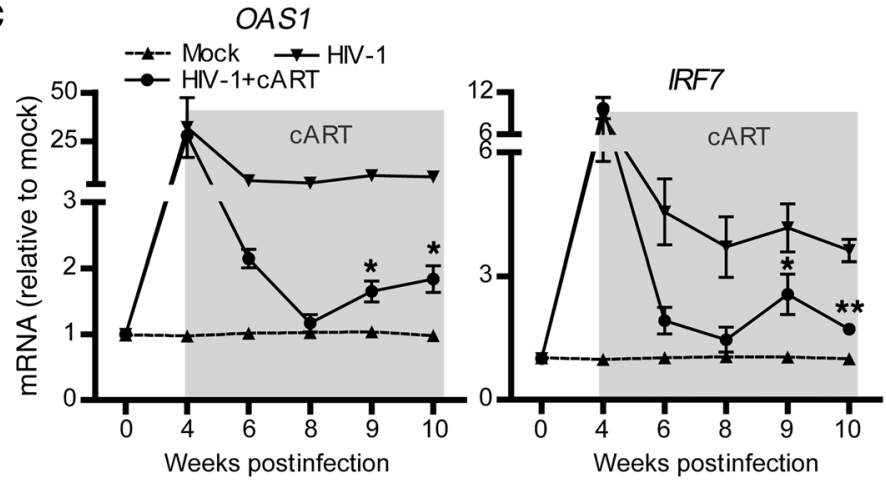

B

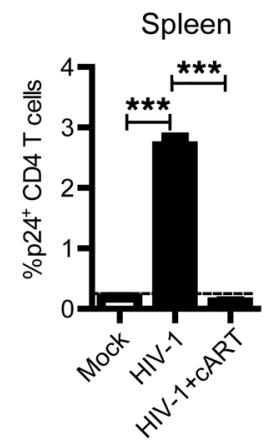

D

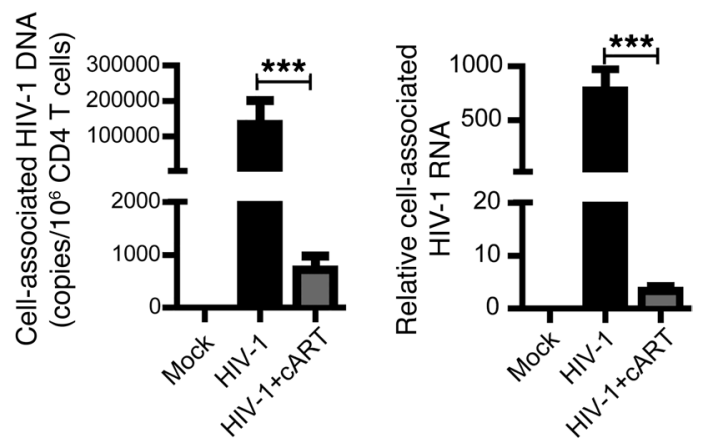

E
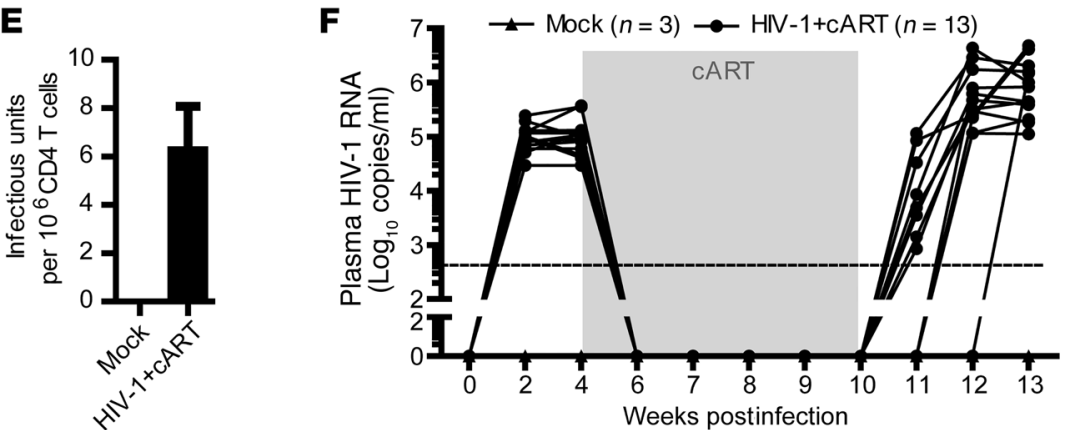

Figure 1. cART efficiently inhibits HIV-1 replication but fails to reverse inflammation and clear HIV-1 reservoirs in hu-mice. (A and B) Hu-mice infected with HIV-1 were treated with cART from 4.5 to 11.5 weeks postinfection (wpi). (A) HIV-1 RNA levels in the plasma of HIV-1-infected ( $n=3$ ) and HIV-1-infected, cART-treated mice $(n=7)$ at indicated time points. (B) Percentage of p24+ CD4 T cells was determined by FACS. Shown are representative data of 3 independent experiments (mock, $n=9$; HIV-1, $n=9$; HIV-1+CART, $n=15$ in total) from $n=4$ (mock), $n=3$ (HIV-1), or $n=7$ (HIV- $1+$ cART) hu-mice per group. (C) Hu-mice infected with HIV-1 were treated with CART from 4 to 10 wpi. Relative mRNA levels of OAS1 and IRF7 in PBMCs are shown at indicated time points. Unpaired, 2-tailed Student's $t$ test was performed to compare between mock and HIV-1+cART group at each single time point. ${ }^{*} P<0.05$, ${ }^{* *} P<0.01$. Shown are combined data from 2 independent experiments with mean values \pm SEM (mock, $n=7$; HIV-1, $n=7$; HIV-1+cART, $n=8$ ). (D) Cell-associated HIV-1 DNA and relative level of cell-associated HIV-1 RNA to human CD4 mRNA in human cells from spleen were quantified by PCR. (E) Replication-competent HIV-1 viruses from spleen were detected by the quantitative virus outgrowth assay. Shown are representative data ( $\mathbf{D}$ and $\mathbf{E})$ from $n=4$ (mock), $n=4$ (HIV-1), and $n=4$ (HIV-1+CART) hu-mice per group of 2 independent experiments. ${ }^{* *} P<0.001$. One-way ANOVA and Bonferroni's post hoc test was performed. (F) Hu-mice infected with HIV-1 were treated with cART from 4 to 10 wpi. CART was discontinued at week 10 . HIV-1 RNA levels in the plasma of each mouse are shown. The broken horizontal line in $\mathbf{F}$ indicates the limit of detection of the assay.

These reports highlight that IFN-I may play important but complex roles in HIV-1 persistent infection and pathogenesis.

In the present study, we developed an antibody against human IFN- $\alpha / \beta$ receptor 1 ( $\alpha$-IFNAR1) to specifically block IFN-I signaling. We found that IFNAR blockade during persistent HIV-1 infection reversed HIV-1-induced immune hyperactivation, rescued anti-HIV-1 immune responses, and reduced the size of HIV-1 reservoirs in lymphoid tissues in the presence of cART. Our results suggest that blocking IFNAR will provide a novel strategy to enhance immune recovery and to reduce HIV-1 reservoirs in those patients with sustained IFN-I signaling during suppressive cART.

\section{Results}

cART efficiently suppresses $H I V-1$ replication but fails to clear $H I V-1$ reservoirs in hu-mice, correlated with low levels of ISG expression. To functionally define the role of IFN-I in HIV-1 persistent infection 
and pathogenesis, we used humanized mice with a functional human immune system (hu-mice) for modeling HIV-1 infection and immunopathogenesis $(26,27)$. We and others have previously reported that persistent HIV-1 infection in hu-mice led to induction of IFN-I signaling, CD4 T cell depletion, aberrant immune activation, and expression of the exhaustion marker PD-1 on $\mathrm{T}$ cells (27-29). As in human patients, cART can efficiently inhibit HIV-1 replication in hu-mice $(30,31)$. We found that plasma viremia decreased to undetectable levels (<400 genome copies/ $\mathrm{ml}$ ) in all HIV-infected hu-mice within 3 weeks after cART treatment (Figure 1A). HIV-1 replication in lymphoid organs was also effectively inhibited by cART (Figure 1B). However, as observed in some patients $(23,24)$, cART failed to completely reduce ISG expression in HIV-1-infected mice to the level of uninfected hu-mice (Figure 1C). HIV-1 reservoirs, measured by cell-associated HIV-1 DNA and RNA (Figure 1D), and cells with infectious HIV-1 (Figure 1E), were still detectable in lymphoid organs of cART-treated hu-mice. As in cART-treated patients, HIV-1 reservoirs persisted stably and virus rebounded rapidly after cART cessation (Figure 1F).

IFNAR blockade during cART-suppressed HIV-1 infection reverses aberrant immune activation. It is reported that abnormally elevated levels of IFN-I signaling and ISG expression persist in some patients even under extensive cART $(23,24)$, which may impede immune recovery and foster viral persistence $(6,25)$. We hypothesized that IFNAR blockade in the presence of cART would reverse hyperimmune or inflammatory activation and facilitate recovery of functional anti-HIV-1 adaptive immune responses, thereby enabling control of cART-resistant HIV-1 reservoirs. To block IFN-I signaling in hu-mice, we developed a monoclonal antibody $(\mathrm{mAb})$ against human IFN- $\alpha / \beta$ receptor 1 ( $\alpha$-IFNAR1) that specifically binds to human IFNAR1 (Supplemental Figure 1A; supplemental material available online with this article; doi:10.1172/ JCI90745DS1) and inhibits human IFN-I activity (Supplemental Figure 1, B and C). The $\alpha$-IFNAR1 mAb neither bound to mouse IFNAR1 (Supplemental Figure 2A) nor blocked mouse IFN-I activity in mouse cells (Supplemental Figure 2B). Furthermore, we showed that the $\alpha$-IFNAR1 mAb effectively blocked ISG induction in vivo in response to the TLR7/8 agonist R848 (resiquimod) in hu-mice (Supplemental Figure 3). Treatment with $\alpha$-IFNAR1 mAb alone affected neither the percentage nor the number of human leukocyte subsets (Supplemental Figure 4, A and B), and administration of $\alpha$-IFNAR1 mAb did not affect the expression of ISGs in splenocytes in hu-mice (Supplemental Figure 4C).

We then analyzed the effect of IFNAR blockade on $\mathrm{T}$ cell activation and functions in the presence of cART treatment in HIV-1-infected hu-mice. We treated HIV-1-infected hu-mice that were fully cART-suppressed with $\alpha$-IFNAR1 mAb for 3 weeks from 7 to 10 weeks postinfection (Figure 2A). As in some cART-treated human patients $(23,24)$, cART failed to completely suppress expression of ISGs (Figure 1C and Figure 2B). In contrast, IFNAR blockade efficiently suppressed HIV-induced ISG expression in cART-treated hu-mice (Figure 2B and Supplemental Figure 4A). HIV-1 persistent infection in hu-mice also induced $\mathrm{T}$ cell depletion, hyperimmune activation, and proliferation as indicated by the expression of the activation marker CD38/HLA-DR and the proliferation marker Ki67 (Figure 2, C-E). Although cART alone significantly rescued the number of human $\mathrm{T}$ cells and total human leukocytes (Figure 2C and Supplemental Figure 5), it only slightly decreased the expression level of CD38/HLA-DR (Figure 2, D and E) and Ki67 on T cells (Figure 2, F and G). Both CD8 and CD4 $\mathrm{T}$ cells from cART-treated hu-mice still expressed significantly higher levels of activation (Figure 2, D and E) and proliferation (Figure 2, F and G) markers in comparison with uninfected hu-mice. Interestingly, IFNAR blockade significantly reversed aberrant CD8 $\mathrm{T}$ cell activation and proliferation in the presence of cART (Figure 2, D-G).

IFNAR blockade reverses the exhaustion phenotype of human $T$ cells and restores anti-HIV-1 T cell function. Despite successful viral inhibition by cART, T cells from patients with poor immune reconstitution sustained higher PD-1 expression (32). We therefore investigated whether IFNAR blockade could reverse expression of PD-1 and other exhaustion markers and rescue HIV-1-specific T cell function in the presence of cART. HIV-1 persistent infection in hu-mice induced both PD-1 and TIM-3 expression on CD8 T cells (Figure 3, A and B). We found that cART alone failed to significantly reduce the expression of PD-1 and TIM-3 (Figure 3, A and B). Interestingly, IFNAR blockade combined with cART completely reduced expression of PD- 1 and TIM- 3 on CD8 T cells (Figure 3 , A and B). Whole transcriptome sequencing of purified human CD8 T cells revealed that cART plus IFNAR blockade also significantly reduced the expression of other $\mathrm{T}$ cell exhaustion markers including CD160, TIGIT (T cell immunoreceptor with Ig and ITIM domains), and BATF (basic leucine transcription factor, ATF-like) (Figure 3C and ref. 33).

We further determined the function of HIV-1-specific T cells after IFNAR blockade. When stimulated with HIV-1 Gag peptide pools ex vivo, both CD8 and CD4 T cells from hu-mice with cART and IFNAR blockade (but not cART alone) produced significantly higher levels of IFN- $\gamma$ and IL-2 (Figure 3, D and E, and Supplemental Figure 6), indicating that IFNAR blockade also rescued the function of HIV-1-specific T cell responses. Taken together, these results indicate that, in the presence of cART, IFNAR blockade can reverse aberrant immune activation and $\mathrm{T}$ cell exhaustion and rescue anti-HIV-1 immune responses.

IFNAR blockade during cART reduces cART-resistant HIV-1 reservoirs. The administration of cART cannot achieve HIV-1 eradication, and virus rebounds quickly after cART discontinuation, owing to the persistence of HIV-1 reservoirs during cART $(34,35)$. It was demonstrated that adaptive immune response contributes to the control of cART-resistant reservoirs $(36,37)$. Thus the improvement of anti-HIV-1 adaptive immune response by therapeutic vaccine or by other immune modulators has been proposed as an immunological strategy for HIV cure $(38,39)$. We postulated that the reversal of immune hyperactivation and the induction of elevated anti-HIV-1 T cell response by blocking of IFNAR might reduce the size of the cART-resistant HIV-1 reservoir and control HIV-1 rebound after cART cessation. We treated HIV-1-infected hu-mice that were fully cART-suppressed with $\alpha$-IFNAR 1 mAb for 3 weeks from 7 to 10 weeks postinfection (Figure 4A). Interestingly, IFNAR blockade, in the presence of cART, led to low blips of HIV-1 replication, which returned to undetectable levels after $\alpha$-IFNAR1 mAb treatment was stopped (Figure 4A). Thus, IFNAR blockade induced activation of HIV-1 
A

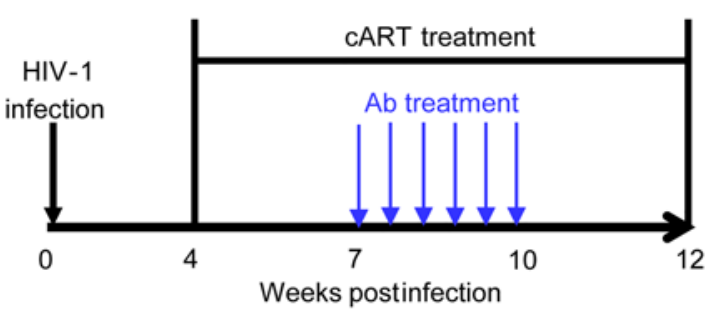

B

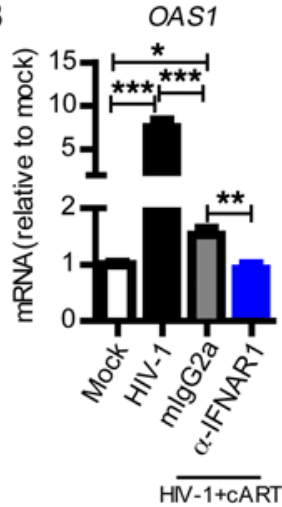

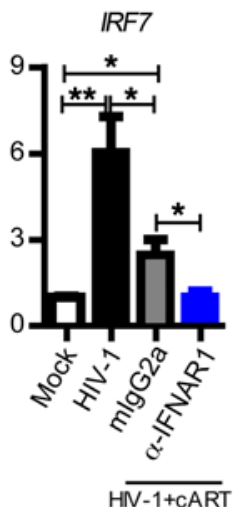

C
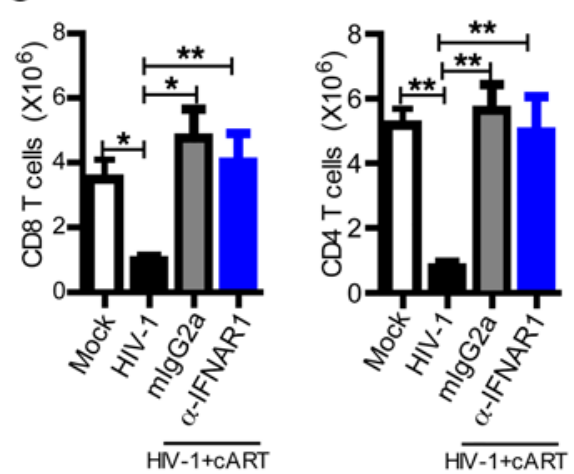

$\mathbf{F}$

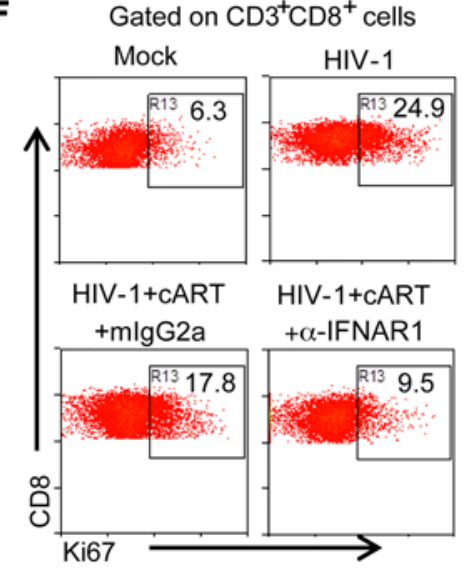

D

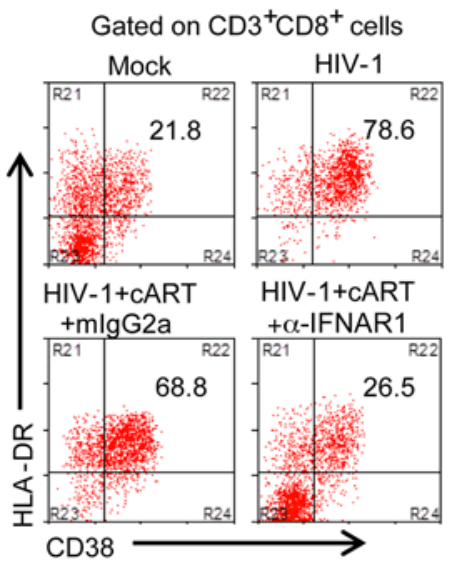

$\mathbf{E}$

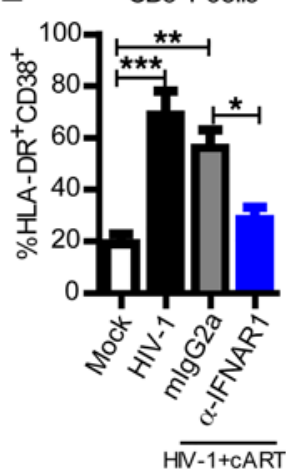

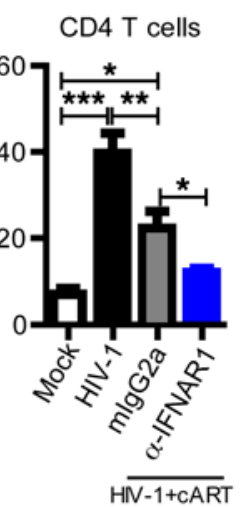

G

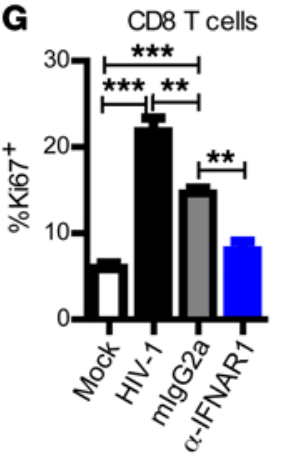

HIV-1+CART

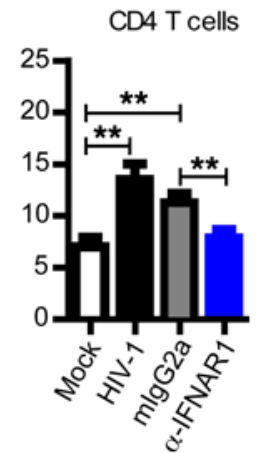

$\mathrm{H} \overline{\mathrm{N}-1+\mathrm{CART}}$

Figure 2. IFNAR blockade during CART-suppressed HIV-1 infection completely reverses aberrant immune activation. (A) Schematic diagram of the experimental design. Hu-mice infected with HIV-1 were treated with CART from 4 to 12 wpi. From 7 to 10 wpi, the cART-treated mice were injected with $\alpha$-IFNAR1 antibody or isotype control mlgG2a antibody twice a week. (B) Relative mRNA levels of OAS1 and IRF7 in PBMCs at 9 wpi. (C) Mice were sacrificed at 12 wpi. Summarized data show numbers of human CD8 and CD4 T cells in spleens. (D) Representative dot plots show percentage HLA-DR+CD38+ of CD8 T cells from spleens. (E) Summarized data show percentage HLA-DR+CD38+ of CD8 and CD4 T cells from spleens. (F) Representative dot plots show percentage Ki67+ ${ }^{+}$of CD8 T cells from spleens. (G) Summarized data show percentage Ki67 ${ }^{+}$of CD8 and CD4 T cells from spleens. Shown are combined data from 2 independent experiments with mean values \pm SEM (mock, $n=7$; HIV-1, $n=7$; HIV-1+cART+mIgG2a, $n=8 ;$ HIV- $1+$ cART $+\alpha-I F N A R 1, n=8$ ). ${ }^{*} P<0.05$, ${ }^{* *} P<0.01,{ }^{* *} P<0.001$, by 1-way ANOVA and Bonferroni's post hoc test.

reservoirs. We speculated that the increase of anti-HIV-1 T cell immune responses by IFNAR blockade would eliminate or control the HIV-1-expressing cells and finally reduce HIV-1 reservoirs. We next analyzed the HIV-1 reservoir size in lymphoid organs 2 weeks after IFNAR blockade. We measured cell-associated HIV-1 DNA and RNA by PCR, and replication-competent HIV-1 by the quantitative virus outgrowth assay. We found that
IFNAR blockade reduced cell-associated HIV-1 DNA 14-fold in the spleen and 4.4-fold in the bone marrow (Figure 4B). Cell-associated HIV-1 RNA was also reduced in both spleen (17.7-fold) and bone marrow (4.4-fold) (Figure 4C). More importantly and consistently, IFNAR blockade significantly reduced the size of replication-competent HIV-1 reservoirs measured by quantitative virus outgrowth assay (Figure 4D). 
A Gated on $\mathrm{CD}^{+} \mathrm{CD}^{+}$cells

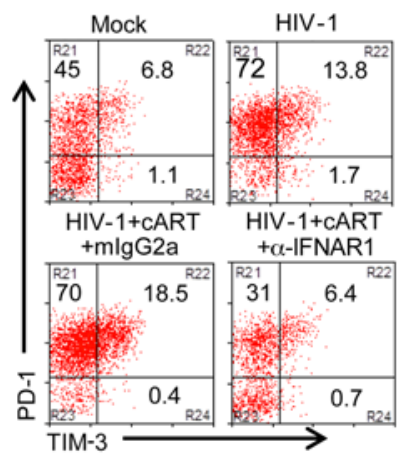

D Gated on $\mathrm{CD}^{+} \mathrm{CD} 8^{+}$cells

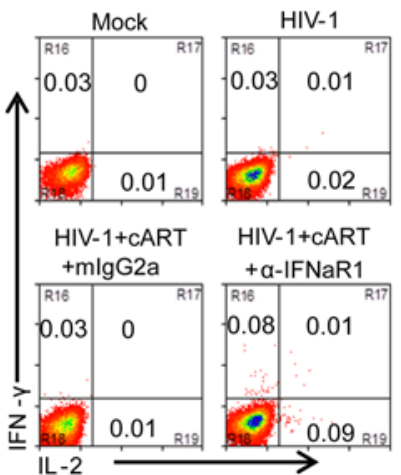

B
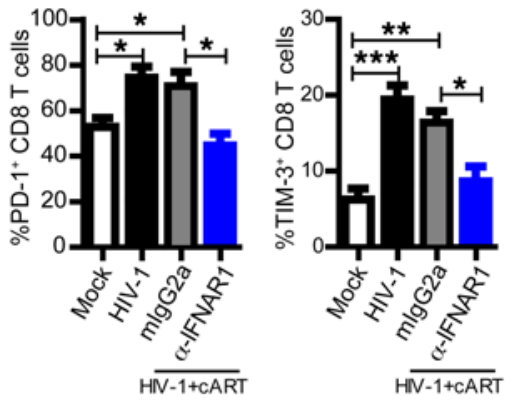

C
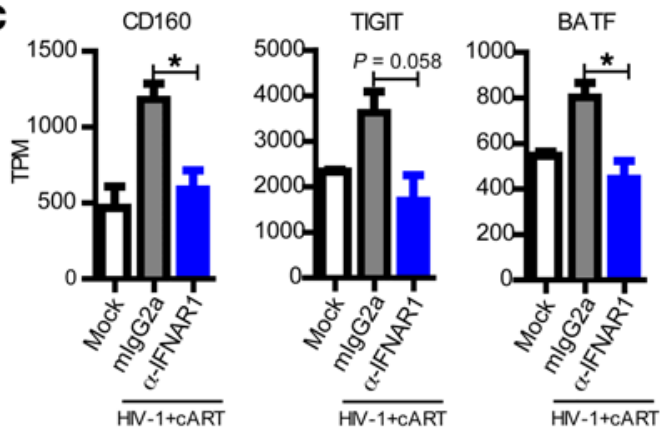

Figure 3. IFNAR blockade during CART-suppressed HIV-1 infection reverses the exhaustion phenotype of CD8 T cells and restores anti-HIV-1 T cell function. Hu-mice were treated as in Figure 2. (A) Representative dot plots show percentage PD-1+ and TIM- $3^{+}$of CD8 T cells from spleens. (B) Summarized data show percentage PD-1+ and TIM-3+ of CD8 T cells from spleens. (C) RNA sequencing was performed with purified CD8 T cells from spleens. Shown is

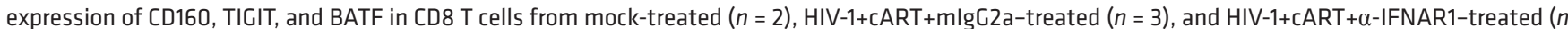
$=3$ ) hu-mice. Transcripts per kilobase million (TPM) indicates the relative abundance of transcripts. Unpaired, 2-tailed Student's $t$ test was performed to compare between groups (C). ( $\mathbf{D}$ and $\mathbf{E}$ ) Splenocytes were stimulated ex vivo with HIV-1 Gag peptide pools for 8 hours (Brefeldin A added at 3 hours) followed by intracellular cytokine staining. Representative dot plots (D) and summarized data (E) show percentages of IFN- $\gamma$ - and IL-2-producing CD8 T cells. Shown are combined data from 2 independent experiments (A, B, D, and E) with mean values \pm SEM (mock, $n=7 ; \mathrm{HIV}-1, n=7 ; \mathrm{HIV}-1+\mathrm{cART}+\mathrm{mlgG} 2 \mathrm{a}$, $n=8$; HIV-1+cART+ $\alpha$-IFNAR1, $n=8$ ). ${ }^{*} P<0.05,{ }^{* *} P<0.01,{ }^{* *} P<0.001$, by 1-way ANOVA and Bonferroni's post hoc test.

IFNAR blockade during cART delays HIV-1 rebound after cART cessation. Finally, we analyzed the effect of IFNAR blockade on HIV-1 rebound after cART discontinuation. Hu-mice with persistent HIV-1 infection received cART, followed by 5 injections of $\alpha$-IFNAR1 mAb after full suppression of HIV-1 (Figure 5A). We stopped cART 2.5 weeks after the last $\alpha$-IFNAR1 mAb injection and monitored virus rebound after cART discontinuation (Figure 5A). One week after cART cessation, HIV-1 rebounded in $56 \%(10 / 18)$ of the control-treated hu-mice, but in none of the hu-mice treated with IFNAR1 blockade (Figure 5, B and C, and Table 1). By the second week, $89 \%(16 / 18)$ of the control-treated hu-mice became HIV-1-positive while only $27 \%$ (3/11) of $\alpha$-IFNAR1 mAb-treated hu-mice showed detectable viremia in the blood (Figure 5, B and C, and Table 1). We also found that cell-associated HIV-1 DNA and RNA in peripheral blood mononuclear cells (PBMCs) was significantly lower in the $\alpha$-IFNAR1 mAb-treated group than in the control antibody-treated group (Figure 5D). By the third week, HIV-1 rebounded in all hu-mice $(18 / 18)$ in the cART-only group and rebounded in $73 \%(8 / 11)$ of the cART plus IFNAR blockade hu-mice (Figure 5, B and C, and Table 1). The viral load in those hu-mice with rebounded HIV-1 at the third week after cART cessation reached the same level between the 2 treatment groups (Figure 5B and Table 1). Based on the findings above, we conclude that IFNAR blockade reduced cART-resistant HIV-1 reservoirs and significantly controlled HIV-1 rebound after cART cessation.

\section{Discussion}

The contribution of IFN-I signaling to HIV-1 persistence during chronic infection is not clearly defined $(5,6)$. Using hu-mice that support HIV-1 persistent infection, we showed that cART efficiently inhibited HIV-1 replication and rescued T cell number in hu-mice with persistent HIV-1 infection. However, low levels of ISG expression, aberrant immune activation, T cell exhaustion, and HIV-1 reservoir persisted stably in cART-treated hu-mice. We demonstrated that IFNAR blockade in cART-treated hu-mice reversed $\mathrm{T}$ cell hyperimmune activation, rescued anti-HIV-1 $\mathrm{T}$ cell immunity, and reduced the size of HIV-1 reservoirs.

Despite efficient suppression of HIV-1 replication with cART, abnormally elevated IFN-I signaling persists in some individuals, which may impede immune recovery and foster viral persistence (23-25). We found here that in hu-mice persistently infected with HIV-1, cART efficiently inhibited viral replication, but failed to completely reverse elevated ISG expression and T cell immune 
A

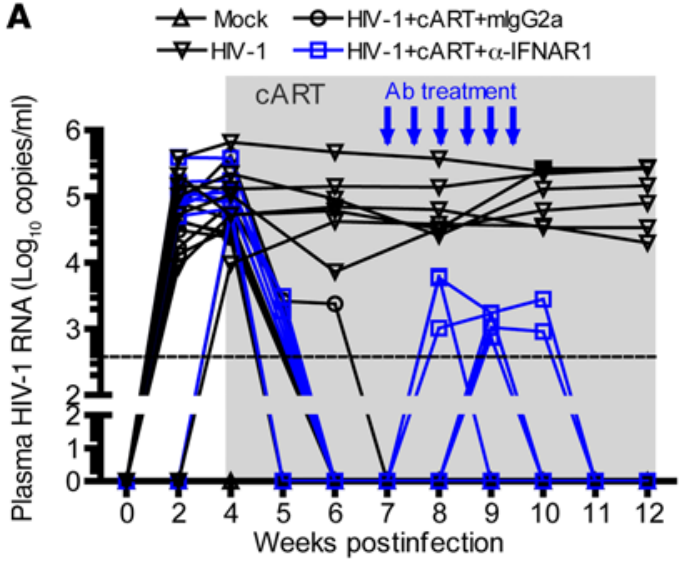

C

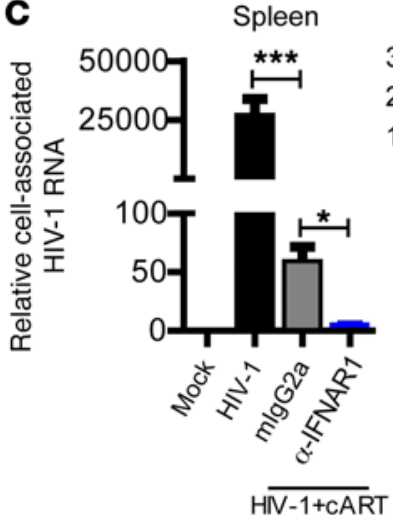

B

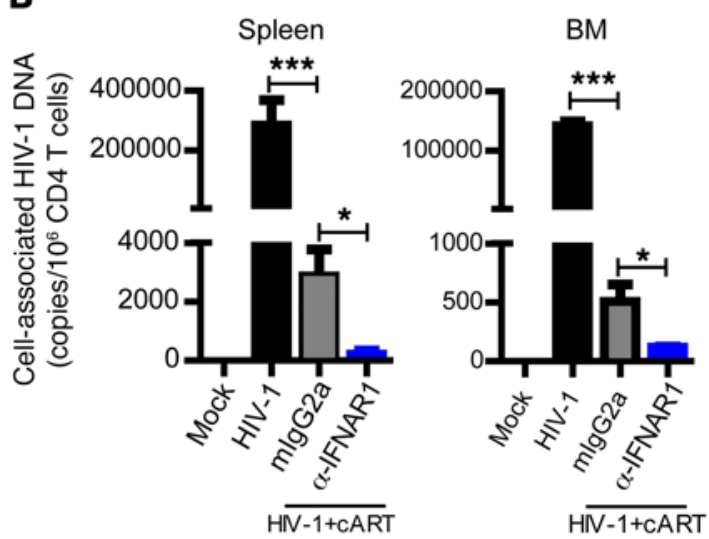

D

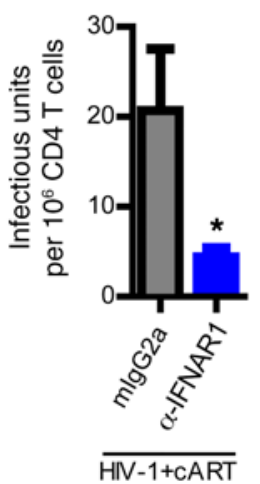

Figure 4. IFNAR blockade during cART reduces cART-resistant HIV-1 reservoirs. Hu-mice infected with HIV-1 were treated with cART from 4 to 12 wpi. From 7 to 10 wpi, the CART-treated mice were injected with $\alpha$-IFNAR1 antibody or isotype control mlgG2a antibody. (A) HIV-1 RNA levels in the plasma. The broken horizontal line indicates the limit of detection (400 copies $/ \mathrm{ml}$ ). (B) Cell-associated HIV-1 DNA in human cells from spleen and bone marrow was quantified by PCR. (C) Relative levels of cell-associated HIV-1 RNA in human cells from spleens and bone marrow were quantified by PCR. (D) Replication-competent HIV-1 viruses from spleens were detected by the quantitative virus outgrowth assay. Shown are combined data from 2 independent experiments with mean values \pm SEM (mock, $n=7$; HIV-1, $n=7$; HIV-1+CART+mlgG2a, $n=8$; HIV-1+CART+ $\alpha$-IFNAR1, $n=8$ ). ${ }^{*} P<0.05,{ }^{* * *} P<0.001$, by 1 -way ANOVA and Bonferroni's post hoc test.

activation and exhaustion. This model thus partially recapitulates the phenotype of those immune nonresponder patients. We showed that blocking IFN-I signaling by using our newly developed anti-IFNAR1 mAb completely inhibited ISG expression, reversed $\mathrm{T}$ cell immune activation and exhaustion, and rescued anti-HIV-1 T cell function. Our results agree with recent findings showing that persistent IFN-I signaling plays a detrimental role during chronic lymphocytic choriomeningitis virus infection and that blockade of IFN-I signaling by IFNAR antibody could reverse $\mathrm{T}$ cell exhaustion and enhance antiviral immune response $(3,4)$. The IFNAR blocking antibody will thus facilitate novel therapeutic development aimed at those difficult-to-treat HIV-1-infected patients with sustained IFN-I signaling during cART $(23,24,40)$.

HIV-1 reservoirs are refractory to antiretroviral therapies (ART) and remain the major barrier to curing $\operatorname{HIV}-1(34,35)$. We report here that IFNAR blockade transiently increased HIV-1 RNA in the blood (viral load "blipping") during cART, indicating that IFN-I signaling contributed to the low replication or latency of the HIV-1 reservoirs. Multiple mechanisms may lead to the reduction of HIV-1 reservoir size after IFNAR blockade during cART. The rescued immune response could target the HIV-1 reservoirs with elevated gene expression and kill the reservoir cells. Other factors, including HIV-1-induced death of reservoir cells and reduced general $\mathrm{T}$ cell activation and proliferation, after IFNAR blockade may also contribute to the reduction of HIV-1 reservoir size. The underlying mechanism of reservoir reduction by IFNAR blockade will be further elucidated in the future. Therefore, blocking IFN-I signaling in cART-treated subjects may provide a novel therapeutic approach for HIV-1 cure (38).

In a recent report, blocking IFN-I signaling with an antagonistic IFN- $\alpha 2$ mutant (IFN-ant) with increased IFNAR2 binding but diminished IFNAR1 binding activity (41) during the acute phase of SIV infection (0-4 weeks postinfection) in rhesus monkeys led to elevated SIV replication and accelerated disease progression (10). Conversely, while preinfection IFN- $\alpha 2$ a administration results in decreased SIV transmission, continued IFN- $\alpha 2$ a treatment appears to induce IFN-I desensitization and decrease antiviral gene expression, resulting in increased SIV replication and accelerated CD4 $\mathrm{T}$ cell loss (10). This study has major differences from our studies in that IFN-I signaling is blocked (or desensitized) only during acute SIV infection. The higher levels of SIV infection probably lead to the accelerated disease progression during the late 
A

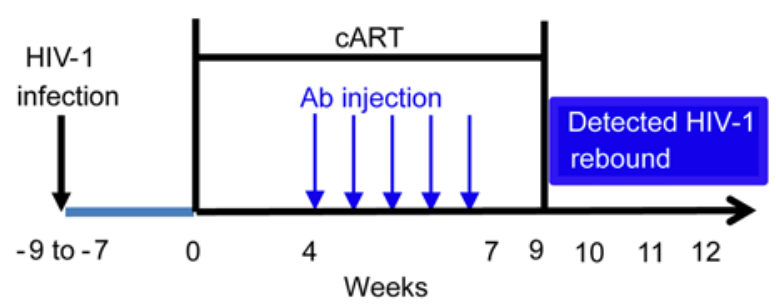

C

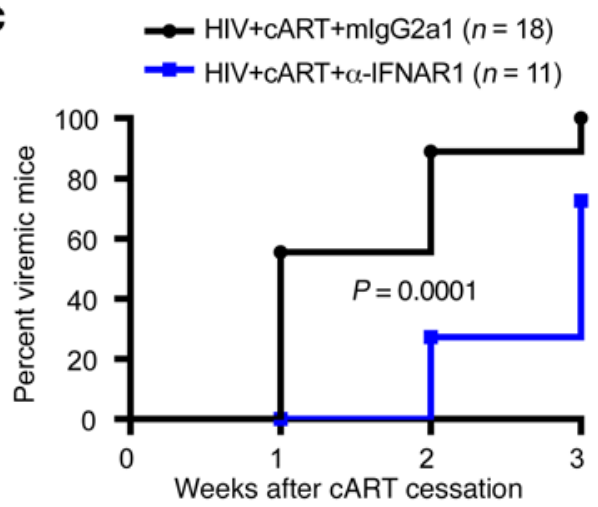

B

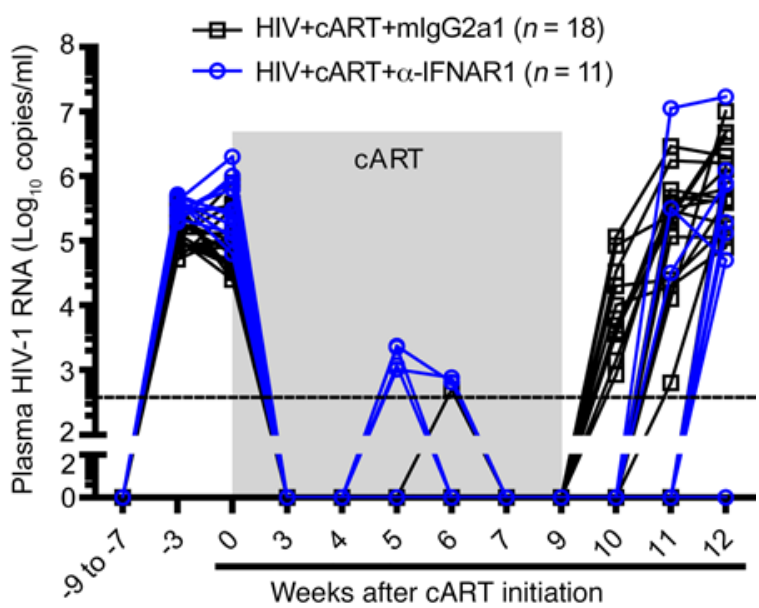

D

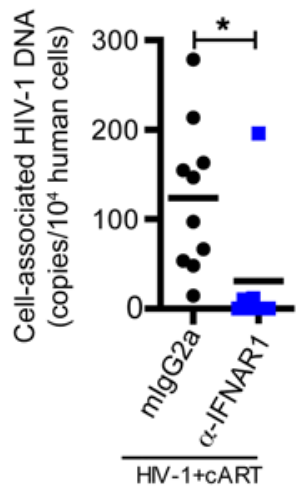

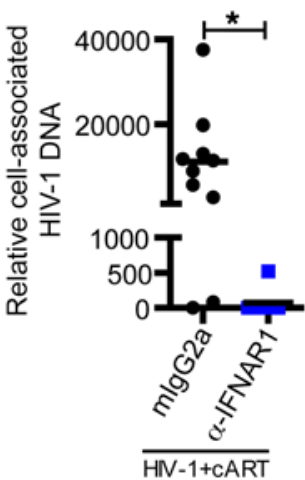

Figure 5. IFNAR blockade during cART delays HIV-1 rebound after cART cessation. (A) Schematic diagram of the experimental design. Hu-mice infected with HIV-1 for 7-9 weeks were treated with cART. The mice were then injected with $\alpha$-IFNAR1 mAb or isotype control mlgC2a antibody 5 times (twice a week) starting from week 4 after cART. cART was maintained for an additional 2.5 weeks after the last antibody treatment. Virus rebound was detected by PCR weekly after cART cessation. (B) Plasma HIV-1 viremia in mice treated with cART plus $\alpha$-IFNAR1 mAb or control mlgG2a. The broken horizontal line indicates the detection limit. (C) Kinetic analysis of HIV-1 rebound after cART cessation. (D) Cell-associated HIV-1 DNA and RNA in PBMCs at 2 weeks after cART cessation. Shown are combined data of 3 independent experiments (B and C) (HIV-1+cART+mlgC2a, $n=18$; HIV-1+cART+ $\alpha$-IFNAR1, $n=11)$ or 2 independent experiments (D) (HIV-1+cART+mlgC2a, $n=10$; HIV-1+CART $+\alpha$-IFNAR1, $n=7$ ) with mean values \pm SEM. ${ }^{*} P<0.05$. Gehan-Breslow-Wilcoxon test (C) or unpaired, 2 -tailed Student's $t$ test (D) was performed.

stage of infection, in the absence of IFN-I blocking. It is generally believed that persistent IFN signaling during chronic infection can lead to general immune suppression (42). Therefore, IFN-I signaling is beneficial during the acute stage to inhibit or prevent virus infection but becomes harmful during the chronic stage of HIV-1 infection. Blocking IFN-I signaling with either the IFNAR mAb or the antagonistic IFN- $\alpha 2$ mutant protein in rhesus monkeys with persistent SIV infection and cART will be of great interest to further clarify these therapeutic strategies.

Several recent reports have shown that the administration of IFN- $\alpha$ in HIV-1-monoinfected or HIV-1/HCV-coinfected patients leads to reduction of cell-associated viral RNA and DNA in the blood cells of a subset of treated patients (18-21). The study by Livio Azzoni et al. (18) reports that long-term administration of IFN- $\alpha$ during and after ART in HIV-1-infected patients leads to suppression of HIV-1 rebound in approximately $40 \%$ of patients, whose PBMC-associated HIV-1 DNA (after 12 weeks with IFN- $\alpha$ only but no ART) is lower in comparison with their PBMCs during ART alone when normalized to their CD4 T cell counts. However, HIV1 reservoirs (cell-associated DNA) were not significantly changed by IFN- $\alpha$ treatment during ART. The administration of IFN- $\alpha$ may induce the migration of activated CD4 T cells into lymphoid organs and subsequently reduction in the peripheral blood (43); thus the reduction may be due to the redistribution of HIV-1 reservoir cells to lymphoid organs induced by IFN- $\alpha$. Interestingly, treatment of HIV-1/HCV-coinfected patients with IFN- $\alpha$ /ribavirin appears to lead to a significant reduction of both CD4 and CD8 T cells $(18,20$, 21 , which is consistent with our previous finding that IFN-I contributes to T cell depletion during chronic HIV-1 infection $(28,44)$. In addition, a low level of HIV-1 replication in the presence of cART may also contribute to the HIV-1 reservoir pool (45). High levels of exogenous IFN-I may inhibit the low-level HIV-1 replication as well as enhance anti-HIV immune responses (46). Therefore, IFN-I signaling may play complex roles during the acute and chronic phases of HIV-1 infection, both inhibiting viral replication and fostering viral persistence by inducing immune dysfunction.

It is important to point out, however, that the human immunity developed in hu-mice is not fully functional as found in immunocompetent hosts $(26,27)$. The restoration of anti-HIV$1 \mathrm{~T}$ cell immune response by IFNAR blockade in hu-mice may not be robust enough to eliminate or fully control the HIV-1 reservoir. Another concern is the xenoreactive graft-versus-host 


\section{Table 1. IFNAR blockade in the presence of CART delays HIV-1 rebound after CART cessation}

\begin{tabular}{|c|c|c|c|c|c|c|c|c|c|c|c|c|}
\hline \multirow[t]{2}{*}{ Donor } & \multirow[t]{2}{*}{ Mouse no. } & \multicolumn{3}{|c|}{$\%$ of cells (preinfection) } & \multirow[t]{2}{*}{ Treatment } & \multicolumn{7}{|c|}{ HIV-1 RNA in plasma $\left(\log _{10}\right)^{D}$} \\
\hline & & $\mathrm{CD}_{4} 5^{\mathrm{A}}$ & $\mathrm{CD}^{\mathrm{B}}$ & $\mathrm{CD}^{\mathrm{C}}$ & & Wo & W4 & W6 & W9 & W10 & W11 & W12 \\
\hline 1 & 1962 & 81.3 & 59.0 & 79.7 & $\mathrm{mlgG} 2 \mathrm{a}$ & 5.0 & ND & ND & ND & ND & ND & 6.2 \\
\hline 1 & 1964 & 74.7 & 68.9 & 82.1 & $\mathrm{mlgG} 2 \mathrm{a}$ & 5.1 & ND & ND & ND & ND & 5.4 & 6.6 \\
\hline 1 & 1972 & 93.9 & 59.2 & 72.9 & $\mathrm{mlgG} 2 \mathrm{a}$ & 4.7 & ND & ND & ND & 5.1 & 6.5 & 6.3 \\
\hline 1 & 1973 & 95.5 & 54.0 & 73.5 & $\mathrm{mlgG} 2 \mathrm{a}$ & 5.1 & ND & ND & ND & 3.6 & 5.8 & 5.6 \\
\hline 1 & 1974 & 90.8 & 48.0 & 79.7 & $\mathrm{mlgG} 2 \mathrm{a}$ & 4.6 & ND & ND & ND & 4.5 & 6.2 & 6.2 \\
\hline 1 & 1975 & 74.9 & 54.2 & 72.2 & $\mathrm{mlgG} 2 \mathrm{a}$ & 4.9 & ND & ND & ND & 4.9 & 5.4 & 6.7 \\
\hline 1 & 1965 & 80.6 & 64.6 & 86.3 & $\alpha$-IFNAR1 & 5.2 & ND & 3.1 & ND & ND & ND & 5.9 \\
\hline 1 & 1966 & 96.8 & 66.4 & 80.5 & $\alpha$-IFNAR1 & 5.0 & ND & 3.4 & ND & ND & 7.1 & 7.2 \\
\hline 1 & 1967 & 62.8 & 47.3 & 70.9 & $\alpha$-IFNAR1 & 5.1 & ND & 0.0 & ND & ND & ND & 6.1 \\
\hline 2 & 1976 & 83.2 & 38.4 & 51.6 & $\mathrm{mlg} \mathrm{G} 2 \mathrm{a}$ & 5.5 & ND & ND & ND & ND & 5.1 & 5.1 \\
\hline 2 & 1978 & 88.1 & 39.6 & 55.2 & mlgG2a & 5.0 & ND & ND & ND & 3.2 & 5.5 & 5.7 \\
\hline 2 & 1979 & 85.7 & 33.4 & 58.3 & $\mathrm{mlgG} 2 \mathrm{a}$ & 5.0 & ND & ND & ND & 3.7 & 5.5 & 5.3 \\
\hline 2 & 1980 & 86.4 & 33.6 & 58.3 & $\mathrm{mlgG} 2 \mathrm{a}$ & 5.6 & ND & ND & ND & 2.9 & 5.7 & 5.6 \\
\hline 2 & 1981 & 80.3 & 35.0 & 32.2 & $\alpha$-IFNAR1 & 5.4 & ND & 3.4 & ND & ND & ND & 5.1 \\
\hline 2 & 1982 & 83.4 & 43.5 & 41.3 & $\alpha$-IFNAR1 & 5.5 & ND & ND & ND & ND & ND & ND \\
\hline 2 & 1984 & 69.4 & 32.2 & 39.4 & $\alpha$-IFNAR1 & 5.4 & ND & 3.0 & ND & ND & ND & ND \\
\hline 2 & 1985 & 77.1 & 29.8 & 57 & $\alpha$-IFNAR1 & 6.0 & ND & ND & ND & ND & ND & 5.3 \\
\hline 3 & 2112 & 15.4 & 54.4 & 74.0 & $\mathrm{mlgG} 2 \mathrm{a}$ & 4.8 & ND & ND & ND & ND & ND & 5.2 \\
\hline 3 & 2113 & 20.9 & 45.6 & 75.6 & $\mathrm{mlg} G 2 \mathrm{a}$ & 4.6 & ND & 2.7 & ND & 3.6 & 5.3 & 6.1 \\
\hline 3 & 2114 & 21.6 & 43.8 & 80.0 & $\mathrm{mlgG} 2 \mathrm{a}$ & 5.9 & ND & ND & ND & ND & 4.1 & 7.0 \\
\hline 3 & 2115 & 10.0 & 59.6 & 63.6 & $\mathrm{mlgG} 2 \mathrm{a}$ & 5.5 & ND & ND & ND & ND & 5.6 & 5.8 \\
\hline 3 & 2176 & 38.1 & 20.6 & 56.9 & $\mathrm{mlgG} 2 \mathrm{a}$ & 4.4 & ND & ND & ND & 4.0 & 4.3 & 4.9 \\
\hline 3 & 2177 & 46.3 & 24.0 & 64.6 & mlgG2a & 4.4 & ND & ND & ND & 4.3 & 4.4 & 5.2 \\
\hline 3 & 2178 & 44.5 & 26.1 & 60.5 & $\mathrm{mlgG} 2 \mathrm{a}$ & 4.7 & ND & ND & ND & ND & 4.3 & 5.6 \\
\hline 3 & 2179 & 37.5 & 25.9 & 62.4 & $\mathrm{mlgG} 2 \mathrm{a}$ & 4.5 & ND & ND & ND & ND & 2.8 & 5.6 \\
\hline 3 & 2124 & 22.5 & 27.5 & 70.1 & $\alpha$-IFNAR1 & 6.3 & ND & ND & ND & ND & ND & 4.7 \\
\hline 3 & 2125 & 4.4 & 45.9 & 62.8 & $\alpha$-IFNAR1 & 4.8 & ND & ND & ND & ND & ND & ND \\
\hline 3 & 2128 & 15.1 & 37.9 & 68.9 & $\alpha$-IFNAR1 & 5.8 & ND & ND & ND & ND & 5.5 & 4.7 \\
\hline 3 & 2174 & 60.9 & 27.9 & 73.7 & $\alpha$-IFNAR1 & 5.9 & ND & ND & ND & ND & 4.5 & 5.9 \\
\hline
\end{tabular}

Hu-mice were treated as in Figure 5E. APercentage of human CD45+ of total cells in PBMCs. ${ }^{\mathrm{B} P e r c e n t a g e}$ of CD3 ${ }^{+}$from human CD45 ${ }^{+}$cells. ${ }^{\mathrm{C}} \mathrm{Percentage}$ of $\mathrm{CD}^{+}$from $\mathrm{CD}^{+}$cells. ${ }^{\circ}$ Weeks after cART treatment. ND, not detectable.

disease (GVHD) in hu-mice cotransplanted with both human hematopoietic stem cells (HSCs) and thymus fragments. GVHD could happen in some long-term-engrafted mice (6-8 months), although the use of hosts lacking the IL-2 common $\gamma$ receptor

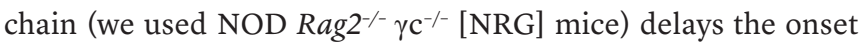
of diseases as reported (47). In our study, most of our experiments were terminated around 5-6 months after human cell transplant. We did not detect significant GVHD symptoms such as conjunctivitis, alopecia, or wasting diseases in the hu-mice during the study (data not shown). Furthermore, we observed similar results when mice humanized with only human HSCs were used, which developed minimal GVHD even more than 9 months after human HSC engraftment (47). Therefore, the key findings in this study are not significantly affected by GVHD. In addition, lymphoid structures such as B cell follicles, which have been identified as HIV-1 sanctuary sites for HIV-1 reservoir cells to escape from CD8 T cell-mediated killing in nonhuman primate models (48) and HIV-infected people (49), are not fully developed in our hu-mice. It is thus possible that HIV-infected cells may be more susceptible to CD8 T cell-mediated control in hu-mice than in primate models or in humans. The findings reported here in hu-mice should accordingly be confirmed with the IFNAR blockade in SIV-infected and cART-treated monkey models before clinical testing in HIV-1 patients.

\section{Methods}

Construction of hu-mice. NRG (NOD Rag2-/- $\mathrm{\gamma c}^{-/-}$) mice were obtained from the Jackson Laboratory. All mice were housed and bred in a specific pathogen-free environment. Humanized NRG mice with a functional human immune system were generated by intrahepatic injection of newborn mice with human fetal liver-derived CD34 ${ }^{+}$hematopoietic progenitor cells as previously reported (28). Humanized BLT (bone marrow/liver/thymus) mice were generated as previously reported (50). Briefly, 6- to 8-week-old NRG mice were sublethally irradiated and anesthetized, and $1-\mathrm{mm}^{3}$ fragments of human fetal thymus were implanted under the kidney capsule. $\mathrm{CD} 4^{+}$hematopoietic progenitor cells purified from fetal liver of the same donor were injected i.v. within 3 hours. Human immune cell engraftment was detected by flow cytometry 12 weeks after transplantation. All animal studies were approved by the University of North Carolina IACUC (ID 14-100). 
HIV-1 infection of hu-mice. The R5 tropic strain of HIV-1 (JR-CSF) was generated by transfection of $293 \mathrm{~T}$ cells with plasmid containing fulllength HIV-1 (JR-CSF) genome. Hu-mice with stable human leukocyte reconstitution were anesthetized and infected with HIV-1 (JR-CSF) (10 ng p24 or 3,000 infectious units per mouse) through retro-orbital injection. Hu-mice infected with 293T supernatant were used as mock control groups. Both male and female mice were used for all the experiments.

Development of anti-IFNAR1 blocking antibody. The mouse cell line L-929 transfected with the human IFNAR1 (extracellular domain and transmembrane domain) expression plasmid mentioned was used as the immunogen for immunization. For each immunization, the WT $\mathrm{BALB} / \mathrm{c}$ female mice were injected i.p. with 5,000,000 immunogen cells with $10 \mu \mathrm{g}$ CpG-1826 as adjuvant. After 5 times immunization, the spleen cells were fused with the mouse myeloma cell line SP2/0. For screening of the clones that could secrete the IFNAR1 binding antibody by flow cytometry, 293T cells transfected with the human IFNAR1 expression plasmid were used. Briefly, the human IFNAR1 expression 293T cell line was firstly incubated with the supernatant of the hybridoma, then incubated with the PE-labeled goat anti-mouse IgG secondary antibody. Then, an IFN-I reporter 293T cell line stably transfected with a mouse $A 2$ promoter-driven EGFP was used to screen antibody clones that could block the human IFNAR1 signaling.

In vitro blocking assay. The IFN-I reporter $293 \mathrm{~T}$ cell line or human PBMCs or mouse splenocytes were preincubated with antibodies for 1 hour in 37 celsius, and the human IFN- $\alpha 2 b$ or mouse IFN- $\alpha$ was added with a final concentration of $5 \mathrm{ng} / \mathrm{ml}$. IFN-I reporter 293T cells were harvested and EGFP expression was analyzed by flow cytometry 24 hours later. The IFN activity after anti-human IFNAR1 treatment relative to samples with IFN- $\alpha 2$ a treatment only was calculated. To detect expression of ISGs in human PBMCs or mouse splenocytes, cells were harvested 4-5 hours later for ISG detection by quantitative real-time PCR. The primers used for the quantitative real-time PCR in the in vitro assay were as follows: human ISG15 (5'-CGCAGATCACCCAGAAGATCG-3' and 5'-TTCGTCGCATTTGTCCACCA-3'), human $M X 2$ (5'-CAGAGGCAGCGGAATCGTAA-3' and 5'-TGAAGCTCTAGCTCGGTGTTC-3'), human EF1A (5'-ATATGGTTCCTGGCAAGCCC-3' and 5'-GTGGGGTGGCAGGTATTAGG-3'), mouse Isg15 (5'-TGGTACAGAACTGCAGCGAG-3' and $5^{\prime}$-AGCCAGAACTGGTCTTCGTG-3'), mouse $M x 2$ (5'-GTGGCAGAGGGAGAATGTCG-3' and 5'-TAAAACAGCATAACCTTTTGCGA-3'), mouse Gapdh (5'-GAGCCAACGGGTCATCT-3' and 5'-GAGGGGCCATCCACAGTCTT-3').

In vivo IFNAR blocking antibody treatments. To confirm the in vivo neutralizing activity of $\alpha$-IFNAR1 mAbs, hu-mice were treated i.p. with $\alpha$-IFNAR1 mAb or mIgG2a as isotype control 6 hours before R848 treatment. HIV-1-infected, cART-treated mice were treated i.p. with IFNAR1 blocking antibodies from 7 to 10 weeks postinfection twice a week with $400 \mu \mathrm{g} /$ mouse at the first injection and $200 \mathrm{~g} / \mathrm{mouse}$ for the following treatments. The same dose of mouse isotype IgG2a control was used in all experiments. Cohorts of mice were randomized into different treatment groups by level of HIV-1 RNA in plasma.

Quantification of mRNA expression in hu-mice by reverse transcriptase PCR. RNA from PBMCs or whole splenocytes from hu-mice was isolated with the RNeasy Plus extraction kit (Qiagen) and converted to cDNA by reverse transcription with random hexamers and SuperScript III First-Strand Synthesis (Invitrogen). cDNA was then subjected to quantitative real-time PCR using human gene-specif- ic primers for ISG15 (5'-CGCAGATCACCCAGAAGATCG-3' and 5'-TTCGTCGCATTTGTCCACCA-3'), OAS1 (5'-TGTCCAAGGTGGTAAAGGGTG-3' and 5'-CCGGCGATTTAACTGATCCTG-3'), MX2 (5'-CAGAGGCAGCGGAATCGTAA-3' and 5'-TGAAGCTCTAGCTCGGTGTTC-3'), and IRF7 (5'-GCTGGACGTGACCATCATGTA-3' and $5^{\prime}$-GGGCCGTATAGGAACGTGC-3'). mRNA from spleen of nonhumanized NRG mice treated with the TLR8 agonist R848 was used as control to test the human specificity of the primers. No signal was detected by real-time PCR when mRNA from spleen of nonhumanized NRG mice treated with R848 was used as control. The ISG expression levels were normalized to mRNA level of human CD45. The result was calculated as a fold change in gene expression relative to mock mice using the $\Delta \Delta \mathrm{Ct}$ method of analysis (51). Briefly, the fold change is calculated by $2^{-\Delta \Delta \mathrm{Ct}} . \Delta \Delta \mathrm{Ct}=(\mathrm{Ct}$ gene of interest $-\mathrm{Ct}$ internal control) sample of treated mouse - (Ct gene of interest - Ct internal control) sample of mock mouse.

Combination antiretroviral therapy. Food formulated with antiretroviral individual drug was prepared as reported with elevated dose modifications (30). In brief, tablets of emtricitabine and tenofovir disoproxil fumarate (Truvada; Gilead Sciences) and raltegravir (Isentress; Merck) were crushed into fine powder and manufactured with TestDiet 5B1Q feed (Modified LabDiet 5058 with 0.12\% amoxicillin) into half-inch irradiated pellets. Final concentrations of drugs in the food were $4,800 \mathrm{mg} / \mathrm{kg}$ raltegravir, $1,560 \mathrm{mg} / \mathrm{kg}$ tenofovir disoproxil, and $1,040 \mathrm{mg} / \mathrm{kg}$ emtricitabine. The estimated daily drug doses were $768 \mathrm{mg} / \mathrm{kg}$ raltegravir, $250 \mathrm{mg} / \mathrm{kg}$ tenofovir disoproxil, and $166 \mathrm{mg} /$ $\mathrm{kg}$ emtricitabine.

Flow cytometry and cell sorting. For surface staining, single-cell suspensions prepared from peripheral blood, spleen, or mesenteric lymph nodes of hu-mice were stained with surface markers and analyzed on a CyAn ADP flow cytometer (Dako). For intracellular staining, cells were first stained with surface markers, and then fixed and permeabilized with Cytofix/Cytoperm buffer (BD Biosciences), followed by intracellular staining. FITC-conjugated anti-human HLA-DR (L243), IFN- $\gamma$ (4S.B3), PE-conjugated anti-human CD38 (HIT2), CD303 (201A), PerCP/Cy5.5-conjugated anti-human CD4 (RPA-T4), PE/ Cy7-conjugated anti-human CD8 (HIT8a), PB-conjugated anti-human CD14 (M5E2), IL-2 (MQ1-17H12), BV421-conjugated anti-human PD-1 (EH12.2H7), APC-conjugated anti-human CD11c (Bu15), and APC/Cy7-conjugated anti-human CD45 (HI30) were purchased from Biolegend. FITC-conjugated anti-HIV-1 p24 was purchased from Beckman Coulter. PE-conjugated anti-human active caspase-3 (C92605) was purchased from BD Pharmingen. Pacific Orange-conjugated anti-mouse CD45 (30-F11), PE/Texas red-conjugated anti-human CD3 (7D6), CD19 (SJ25-C1), and LIVE/DEAD Fixable Yellow Dead Cell Stain Kit were purchased from Invitrogen. Data were analyzed using Summit 4.3 software (Dako).

For CD8 T cell sorting, after staining with viability dye and surface markers (anti-hCD45, -mCD45, -hCD3, -hCD4, -hCD8, -hCD11c, -hCD14, -hCD123), CD8 T cells (hCD45 $\left.{ }^{+} \mathrm{mCD}_{4} 5^{-h C D} 3^{+} \mathrm{hCD} 8^{+} \mathrm{hCD} 4^{-}\right)$ were sorted on a BD FACSAria II using a 70-mm nozzle and collected into Falcon round-bottom polypropylene tubes containing RPMI $1640 / 10 \%$ FBS. The purity of sorted CD8 T cells was above $99 \%$.

$T$ cell stimulation and intracellular cytokine staining. For nonspecific stimulation, splenocytes from hu-mice were stimulated ex vivo with PMA (phorbol 12-myristate 13 -acetate) $(50 \mathrm{ng} / \mathrm{ml})$ and ionomycin $(1 \mu \mathrm{M})$ (Sigma-Aldrich) for 4 hours in the presence of brefeldin A (Biolegend). 
For antigen-specific stimulation, splenocytes from hu-mice were stimulated ex vivo with peptide pools ( $2 \mu \mathrm{g} / \mathrm{ml}$ for each peptide) for HIV-1 GAG protein [PepMix HIV (GAG) Ultra; JPT Innovation Peptide Solutions] for 3 hours without brefeldin A and then 5 hours in the presence of brefeldin A. Cells were then fixed and permeabilized with Cytofix/Cytoperm buffer (BD Biosciences), and intracellular staining was then performed.

HIV-1 genomic RNA detection in plasma. HIV-1 RNA was purified from the plasma with the QIAampkit Viral RNA Mini Kit. The RNA was then reverse-transcribed and quantitatively detected by real-time PCR using the TaqMan Fast Virus 1-Step PCR kit (Thermo Fisher Scientific). The primers used for detecting the HIV gag gene were 5'-GGTGCGAGAGCGTCAGTATTAAG-3' and 5'-AGCTCCCTGCTTGCCCATA-3'. The probe (FAM-AAAATTCGGTTAAGGCCAGGGGGAAAGAA-QSY7) used for detection was ordered from Applied Biosystems, and the reactions were set up following the manufacturer's guidelines and were run on the QuantStudio 6 Flex PCR system (Applied Biosystems). The detection limit of the real-time PCR reaction is 4 copies per reaction. Accordingly, due to the relatively small volume of each bleeding in mice (around 50-100 $\mu$ total blood), the limit of detection of the assay is 400 copies/ml plasma. We set the copy numbers that were below the detectable limit as 1 .

Cell-associated HIV-1 DNA detection. To measure total cell-associated HIV-1 DNA, nucleic acid was extracted from spleen and bone marrow cells using the DNeasy mini kit (Qiagen). HIV-1 DNA was quantified by real-time PCR. DNA from serial dilutions of ACH2 cells, which contain 1 copy of HIV genome in each cell, was used to generate a standard curve.

Cell-associated HIV-1 RNA detection. To measure total cell-associated HIV-1 RNA, nucleic acid was extracted from spleen or bone marrow cells using the RNeasy plus mini kit (Qiagen). HIV-1 RNA was detected as described above. The HIV-1 RNA expression levels were normalized to human CD4 mRNA (5'-GGGCTTCCTCCTCCAAGTCTT-3' and CCGCTTCGAGACCTTTGC) controls, and the result was calculated as fold change in gene expression.

Viral outgrowth assay. Viral outgrowth assay was performed as reported (52). Serial dilutions of human cells from splenocytes of hu-mice $\left(1 \times 10^{6}, 2 \times 10^{5}, 4 \times 10^{4}\right.$ human cells $)$ were stimulated with phytohemagglutinin $(2 \mu \mathrm{g} / \mathrm{ml})$ and IL-2 (100 U/ml) for 24 hours. MOLT4/ CCR 5 cells were added on day 2 to enhance the survival of the cultured cells as well as to support and facilitate further HIV-1 replication. Culture medium containing IL-2 (NIH AIDS Reagent Program; https://www. aidsreagent.org/) and T cell growth factor (homemade as described in the standard protocol) was replaced on days 5 and 9. After 7 and 14 days of culture, supernatant from each well was harvested and HIV-1 reverse transcriptase quantitative PCR was performed to score viral outgrowth. Estimated frequencies of cells with replication-competent HIV-1 were calculated using limiting dilution analysis.

RNA sequencing. Purified human CD8 T cells from spleens of hu-mice as described above were used to prepare RNA. The cDNA was prepared using SMART Seq v4 Ultra Low RNA-Seq kit for 48 reactions (Clontech). A Nextera kit was used for library construction, and sequencing was performed on Illumina HiSeq2500v4 with paired-end sequencing for 50 cycles. Sequencing data FASTQ files for samples were processed in Salmon workflow in a Linux server operating system to output gene-level abundance estimates and statistical inference as gene-level raw counts. Those raw counts for samples were input into the edgeR program (Bioconductor) for differential gene expression analysis.

Statistics. Statistical analyses were performed using GraphPad Prism 5.0 software (GraphPad Software). Experiments were analyzed by 2-tailed Student's $t$ test, or by 1-way ANOVA and Bonferroni's post hoc test or Gehan-Breslow-Wilcoxon test, according to the assumptions of the test, as indicated in the figure legends for each experiment. ${ }^{*} P<0.05$, ${ }^{* *} P<0.01,{ }^{* *} P<0.001$. All the data with error bars are presented as mean values \pm SEM. A $P$ value less than 0.05 was considered significant.

Study approval. Human fetal liver and thymus tissues (gestational age 16-20 weeks) were obtained from elective or medically indicated termination of pregnancy through a nonprofit intermediary working with outpatient clinics (Advanced Bioscience Resources, Alameda, California, USA). Informed consent of the maternal donors was obtained in all cases, under regulation governing the clinic. The project was reviewed by the University of North Carolina at Chapel Hill Office of Human Research Ethics, which has determined that this submission does not constitute human subjects research as defined under federal regulations [45 CFR 46.102 (d or f) and 21 CFR 56.102(c)(e)(l)]. All animal studies were approved by the University of North Carolina IACUC.

\section{Author contributions}

LC, LS, and LZ conceived and designed the study. LC, JM, JL, DL, GL, FL, QZ, HY, FY, CY, LCT, and ZH performed experiments. LC, LS, and LZ analyzed and interpreted the data and prepared the manuscript.

\section{Acknowledgments}

We thank L. Chi and Y. Wu for technical support; the Lineberger Comprehensive Cancer Center cores and the University of North Carolina Flow Cytometry Core, Division of Laboratory Animal Medicine, and Center for AIDS Research for support; J.M. McCune for discussion and editing of the manuscript; and Ron Swanstrom and G. Silvestri for discussion. This study was supported in part by NIH grants R01AI095097 and R01AI080432 to LS and a grant from the Beijing Municipal Science and Technology Commission (SCW 2014-09) to LZ.

Address correspondence to: Lishan Su, Room 5208, Masico Hall, 125 Mason Farm Road, Chapel Hill, North Carolina 27514, USA. Phone: 919.966.6654; E-mail: lsu@med.unc.edu. Or to: Liguo Zhang, 15 Datun Road, Chaoyang District, Beijing, China, 100101. Phone: 010.64862568; E-mail: liguozhang@ibp.ac.cn.
1. Zuniga EI, Hahm B, Oldstone MB. Type I interferon during viral infections: multiple triggers for a multifunctional mediator. Curr Top Microbiol Immunol. 2007;316:337-357.

2. Schoggins JW, et al. A diverse range of gene products are effectors of the type I interferon antiviral response. Nature. 2011;472(7344):481-485.
3. Wilson EB, et al. Blockade of chronic type I interferon signaling to control persistent LCMV infection. Science. 2013;340(6129):202-207.

4. Teijaro JR, et al. Persistent LCMV infection is controlled by blockade of type I interferon signaling. Science. 2013;340(6129):207-211.

5. Doyle T, Goujon C, Malim MH. HIV-1 and inter- ferons: who's interfering with whom? Nat Rev Microbiol. 2015;13(7):403-413.

6. Bosinger SE, Utay NS. Type I interferon: understanding its role in HIV pathogenesis and therapy. Curr HIV/AIDS Rep. 2015;12(1):41-53.

7. Stacey AR, et al. Induction of a striking systemic cytokine cascade prior to peak viremia in acute 
human immunodeficiency virus type 1 infection, in contrast to more modest and delayed responses in acute hepatitis B and $\mathrm{C}$ virus infections. J Virol. 2009;83(8):3719-3733.

8. Hardy GA, et al. Interferon- $\alpha$ is the primary plasma type-I IFN in HIV-1 infection and correlates with immune activation and disease markers. PLoS One. 2013;8(2):e56527.

9. Lavender KJ, et al. Interferon $\alpha$ subtype-specific suppression of HIV-1 infection in vivo. JVirol. 2016;90(13):6001-6013.

10. Sandler NG, et al. Type I interferon responses in rhesus macaques prevent SIV infection and slow disease progression. Nature. 2014;511(7511):601-605.

11. Jacquelin B, et al. Nonpathogenic SIV infection of African green monkeys induces a strong but rapidly controlled type I IFN response. J Clin Invest. 2009;119(12):3544-3555.

12. Bosinger SE, et al. Global genomic analysis reveals rapid control of a robust innate response in SIV-infected sooty mangabeys. JClin Invest. 2009;119(12):3556-3572.

13. Harris LD, et al. Downregulation of robust acute type I interferon responses distinguishes nonpathogenic simian immunodeficiency virus (SIV) infection of natural hosts from pathogenic SIV infection of rhesus macaques. JVirol. 2010;84(15):7886-7891.

14. Favre D, et al. Critical loss of the balance between Th17 and T regulatory cell populations in pathogenic SIV infection. PLoS Pathog. 2009;5(2):e1000295.

15. Rotger M, et al. Genome-wide mRNA expression correlates of viral control in CD4 ${ }^{+} \mathrm{T}$-cells from HIV-1-infected individuals. PLoS Pathog. 2010;6(2):e1000781.

16. Hyrcza MD, et al. Distinct transcriptional profiles in ex vivo $\mathrm{CD} 4^{+}$and $\mathrm{CD} 8^{+} \mathrm{T}$ cells are established early in human immunodeficiency virus type 1 infection and are characterized by a chronic interferon response as well as extensive transcriptional changes in CD8+ T cells. JVirol. 2007;81(7):3477-3486.

17. Sedaghat $\mathrm{AR}$, et al. Chronic $\mathrm{CD} 4^{+} \mathrm{T}$-cell activation and depletion in human immunodeficiency virus type 1 infection: type I interferonmediated disruption of T-cell dynamics. J Virol. 2008;82(4):1870-1883.

18. Azzoni L, et al. Pegylated interferon alfa-2a monotherapy results in suppression of HIV type 1 replication and decreased cell-associated HIV DNA integration. J Infect Dis. 2013;207(2):213-222.

19. Sun $H$, et al. Hepatitis $C$ therapy with interferon- $\alpha$ and ribavirin reduces $\mathrm{CD} 4 \mathrm{~T}$-cell-associated HIV-1 DNA in HIV-1/hepatitis C virus-coinfected patients. J Infect Dis. 2014;209(9):1315-1320.

20. Jiao YM, et al. Hepatitis $C$ therapy with interferon- $\alpha$ and ribavirin reduces the $\mathrm{CD} 4$ cell count and the total, 2LTR circular and integrated HIV-1 DNA in HIV/HCV co-infected patients. Antiviral
Res. 2015;118:118-122.

21. Morón-López S, et al. Short-term treatment with interferon alfa diminishes expression of HIV-1 and reduces $\mathrm{CD} 4^{+} \mathrm{T}$-cell activation in patients coinfected with HIV and hepatitis $C$ virus and receiving antiretroviral therapy. J Infect Dis. 2016;213(6):1008-1012.

22. Manion $\mathrm{M}$, et al. Interferon- $\alpha$ administration enhances $\mathrm{CD} 8+\mathrm{T}$ cell activation in HIV infection. PLoS One. 2012;7(1):e30306.

23. Fernandez $\mathrm{S}$, et al. $\mathrm{CD} 4^{+} \mathrm{T}$-cell deficiency in HIV patients responding to antiretroviral therapy is associated with increased expression of interferon-stimulated genes in $\mathrm{CD} 4^{+} \mathrm{T}$ cells. J Infect Dis. 2011;204(12):1927-1935.

24. Dunham RM, et al. Discordance between peripheral and colonic markers of inflammation during suppressive ART. J Acquir Immune Defic Syndr. 2014;65(2):133-141.

25. Deeks SG. HIV infection, inflammation, immunosenescence, and aging. Annu Rev Med. 2011;62:141-155.

26. Shultz LD, Brehm MA, Garcia-Martinez JV, Greiner DL. Humanized mice for immune system investigation: progress, promise and challenges. Nat Rev Immunol. 2012;12(11):786-798.

27. Zhang L, Su L. HIV-1 immunopathogenesis in humanized mouse models. Cell Mol Immunol. 2012;9(3):237-244.

28. Li G, et al. Plasmacytoid dendritic cells suppress HIV-1 replication but contribute to HIV-1 induced immunopathogenesis in humanized mice. PLoS Pathog. 2014;10(7):e1004291.

29. Seung E, Dudek TE, Allen TM, Freeman GJ, Luster AD, Tager AM. PD-1 blockade in chronically HIV-1-infected humanized mice suppresses viral loads. PLoS One. 2013;8(10):e77780.

30. Halper-Stromberg A, et al. Broadly neutralizing antibodies and viral inducers decrease rebound from HIV-1 latent reservoirs in humanized mice. Cell. 2014;158(5):989-999.

31. Choudhary SK, Archin NM, Cheema M, Dahl NP, Garcia JV, Margolis DM. Latent HIV-1 infection of resting $\mathrm{CD} 4^{+} \mathrm{T}$ cells in the humanized $\mathrm{Rag} 2^{-/-} \mathrm{cc}^{-/}$ mouse. JVirol. 2012;86(1):114-120.

32. Grabmeier-Pfistershammer K, Steinberger P, Rieger A, Leitner J, Kohrgruber N. Identification of PD-1 as a unique marker for failing immune reconstitution in HIV-1-infected patients on treatment. JAcquir Immune Defic Syndr. 2011;56(2):118-124.

33. Wherry EJ, Kurachi M. Molecular and cellular insights into T cell exhaustion. Nat Rev Immunol. 2015;15(8):486-499.

34. Katlama C, et al. Barriers to a cure for HIV: new ways to target and eradicate HIV-1 reservoirs. Lancet. 2013;381(9883):2109-2117.

35. Archin NM, Sung JM, Garrido C, Soriano-Sarabia N, Margolis DM. Eradicating HIV-1 infection: seeking to clear a persistent pathogen. Nat Rev Microbiol.2014;12(11):750-764.
36. Deng K, et al. Broad CTL response is required to clear latent HIV-1 due to dominance of escape mutations. Nature. 2015;517(7534):381-385.

37. Shan L, et al. Stimulation of HIV-1-specific cytolytic $\mathrm{T}$ lymphocytes facilitates elimination of latent viral reservoir after virus reactivation. Immunity. 2012;36(3):491-501.

38. Barouch DH, Deeks SG. Immunologic strategies for HIV-1 remission and eradication. Science. 2014;345(6193):169-174.

39. Siliciano RF. Opening Fronts in HIV Vaccine Development: Targeting reservoirs to clear and cure. Nat Med. 2014;20(5):480-481.

40. Zhang Z, et al. Safety and immunological responses to human mesenchymal stem cell therapy in difficult-to-treat HIV-1-infected patients. AIDS. 2013;27(8):1283-1293.

41. Levin D, et al. Multifaceted activities of type I interferon are revealed by a receptor antagonist. Sci Signal. 2014;7(327):ra50.

42. Crouse J, Kalinke U, Oxenius A. Regulation of antiviral $\mathrm{T}$ cell responses by type I interferons. Nat Rev Immunol. 2015;15(4):231-242.

43. Massanella M, et al. Changes in T-cell subsets in HIV-HCV-coinfected patients during pegylated interferon- $\alpha 2$ a plus ribavirin treatment. Antivir Ther (Lond). 2010;15(3):333-342.

44. Zhang Z, et al. Plasmacytoid dendritic cells promote HIV-1-induced group 3 innate lymphoid cell depletion. JClin Invest. 2015;125(9):3692-3703.

45. Lorenzo-Redondo R, et al. Persistent HIV-1 replication maintains the tissue reservoir during therapy. Nature. 2016;530(7588):51-56.

46. Tomescu C, Mavilio D, Montaner LJ. Lysis of HIV-1-infected autologous $\mathrm{CD}^{+}$primary $\mathrm{T}$ cells by interferon- $\alpha$-activated NK cells requires NKp46 and NKG2D. AIDS. 2015;29(14):1767-1773.

47. Greenblatt MB, et al. Graft versus host disease in the bone marrow, liver and thymus humanized mouse model. PLoS One. 2012;7(9):e44664.

48. Fukazawa $Y$, et al. B cell follicle sanctuary permits persistent productive simian immunodeficiency virus infection in elite controllers. Nat Med. 2015;21(2):132-139.

49. Perreau M, et al. Follicular helper T cells serve as the major CD4 T cell compartment for HIV-1 infection, replication, and production. JExp Med 2013;210(1):143-156

50. Namikawa R, Kaneshima H, Lieberman M, Weissman IL, McCune JM. Infection of the SCID-hu mouse by HIV-1. Science. 1988;242(4886):1684-1686.

51. Schmittgen TD, Livak KJ. Analyzing real-time PCR data by the comparative $\mathrm{C}(\mathrm{T})$ method. Nat Protoc. 2008;3(6):1101-1108.

52. Laird GM, Rosenbloom DI, Lai J, Siliciano RF, Siliciano JD. Measuring the frequency of latent HIV-1 in resting $\mathrm{CD} 4^{+} \mathrm{T}$ cells using a limiting dilution coculture assay. Methods Mol Biol. 2016;1354:239-253. 